\title{
A Simple Home-Based Lifestyle Intervention Program to Improve Cardiac Autonomic Regulation in Patients with Increased Cardiometabolic Risk
}

\author{
Daniela Lucini ${ }^{1,2, *}$, Mara Malacarne ${ }^{1,2}$, Wolfgang Gatzemeier ${ }^{3}$ and Massimo Pagani ${ }^{1}$ \\ 1 BIOMETRA Department, University of Milan, 20122 Milan, Italy; mara.malacarne@unimi.it (M.M.); \\ Massimo.paganiz@gmail.com (M.P.) \\ 2 Exercise Medicine Unit, Humanitas Clinical and Research Center, 20089 Rozzano, Italy \\ 3 Cancer Center, Humanitas Clinical and Research Center, 20089 Rozzano, Italy; \\ wolfgang.gatzemeier@humanitas.it \\ * Correspondence: daniela.lucini@unimi.it; Tel.: +39-02-82247449
}

Received: 22 July 2020; Accepted: 15 September 2020; Published: 17 September 2020

\begin{abstract}
Lifestyle modification programs (LMP) represent a new approach to cardiometabolic/ oncologic risk reduction. Successful LMP in clinical practice must be feasible, cost effective, efficacious and consider home-based exercise. Likewise, multiple mechanisms implied in cardiometabolic risk reduction such as cardiac autonomic regulation (CAR) should be easily evidenced, in spite of the computational complexity involved. This goal could be facilitated by employing novel, friendlier, simpler techniques, such as the Autonomic Nervous System Index (ANSI), which can be treated as a proxy of CAR. In this observational study, we introduce a simple LMP (based on cognitive behavioral strategies and patient-tailored prescription of nutrition and home-based exercise, managed by a single physician) into the currently existing clinical practice of secondary cardiometabolic prevention. In 26 subjects, we assessed CAR (autoregressive spectral analysis of cardiovascular variabilities), body mass composition (bioelectrical impedance analysis) and stress perception (questionnaires). After LMP, ANSI and lipid profile were improved; \% of fat mass, waist circumference and stress perception were reduced. We conclude that this preliminary, proof of concept study provides significant evidence in favor of the hypothesis that it is possible to introduce a convenient, cost effective LMP into the currently existing clinical practice of secondary cardiometabolic prevention. Findings suggest a successful comprehensive behavioral change, possibly facilitated by the simplified approach employed in this study, capable of improving cardiac autonomic regulation in addition to body mass composition and stress perception.
\end{abstract}

Keywords: autonomic nervous system; prevention; exercise; home-based intervention; nutrition; heart rate variability

\section{Introduction}

Lifestyle medicine [1] represents a new approach to cardiometabolic/oncologic risk reduction, aiming not only to reduce risk factors but primarily to ameliorate healthy lifestyles such as improvement in physical activity habits, healthy nutrition, cessation of smoking and stress management strategies. Lifestyle modifications can potentially prevent $70 \%$ to $90 \%$ of cardiometabolic diseases and $40 \%$ to $70 \%$ of cancer-related deaths [2] and are now considered mandatory in all international guidelines to manage chronic non-communicable diseases [3-5].

There are multiple approaches to lifestyle modification [6], characterized by differences in required levels of expertise of the involved professionals, lengths of allotted time, employed protocols, operational context, clinical and psychological characteristics of subjects and specific preventive goals. 
As a result, efficacy varies considerably across different approaches to intervention [6]. The programs that appear to be most effective, particularly in secondary prevention, are characterized by multifactorial interventions and are designed for individuals or groups according to specific characteristics and needs [6-10]. Unfortunately, these complex programs are expensive, are time consuming and require that the different healthcare professionals work in truly collaborative and goal-oriented teams in order to achieve positive results [10-14]. On the contrary, simple referral to other healthcare professionals without joint responsibility often results in a time consuming, expensive and rather ineffective approach $[10,13,15]$. Notably, this strategy is frequently present in clinical contexts outside research programs, representing paradoxically a barrier to a successful result. On the other hand, the possibility of accommodating all elements of lifestyle modification programs into relatively few encounters with a single trained physician could be advantageous to hopeful patients and to interested institutions, because adequate results could be obtained without increasing cost or time. Moreover, home-based exercise programs are welcomed by patients, being convenient from an economical point of view and representing a bridge toward introducing exercise into everyday routine.

In line with this reasoning, and particularly in the case of long-term conditions, the concept that medical and functional aspects could be synthetized with everyday, normal clinical practice was advocated years ago in a psychosomatic context by BT Engel [16] and more recently from a systems perspective by AC Ahn et al. [17].

Accordingly, with proper training, physicians can learn how to produce therapeutic behavioral responses (such as increasing physical activity or reducing weight).

It is also important to recall that an LMP should be considered "efficacious" only if its application results in improvement of the underlying pathogenetic mechanisms. They range from a betterment of immunological and hormonal profiles to improvement of hemodynamics. Improvements in autonomic nervous system (ANS) regulation may also play an additional, little recognized, beneficial role [18] generating a "risk factors gap" above and beyond usual treatments. Several chronic diseases such as arterial hypertension, ischemic heart disease, diabetes and even cancer are characterized by ANS impairment [19-22], which may be reversed by exercise training and/or healthy nutrition programs [18,22-25]. Subjects with overweight and obesity are characterized both at an adult and young age by well recognized ANS dysfunctions [23]; breast cancer patients show an ANS impairment also as a consequence of adjuvant therapies [26]. Conversely, weight management programs are associated with a combination of improved vagal function and reduced sympathetic activity [18,22-25].

In this preliminary, proof of concept, study we consider a group of hopeful subjects (subjects who were willing to come back periodically to our clinic for a long follow-up period and periodical evaluation) characterized by an increased cardiometabolic risk as occurs in patients with overweight, metabolic syndrome or in breast cancer survivors. We hypothesize that it would be feasible to introduce a convenient lifestyle modification program (LMP) $[1,3,5-7,10,13]$ into the currently existing clinical practice of secondary cardiometabolic prevention (avoiding increases in allotted time and costs) considering home-based exercise protocols individually prescribed. We also tested whether, in addition to expected favorable changes (such as the betterment of metabolic parameters), cardiac autonomic regulation, as assessed by a simple unitary autonomic proxy (ANSI) [27], would be improved.

\section{Materials and Methods}

In this observational, proof of concept study, we consider a group of 26 metabolic syndrome ambulant patients (21 of them were breast cancer survivors) who were referred to the Exercise Medicine Unit (Humanitas Research Hospital, Rozzano, Italy) by their physician in order to reduce cardiometabolic risk. Intervention program was followed a personalized approach intended to reduce cardiometabolic (hence, in part, also oncologic) risk factors, combining cognitive behavioral strategies-in particular, considering motivational interviewing models $[1,28,29]$ with patient-tailored prescription of exercise and nutrition as typical of a lifestyle medicine approach [1], according to recent guidelines $[3-7,10,13,30,31]$. Eligibility criteria included the absence of acute conditions (within the 
past three months) or the presence of chronic diseases such as diabetes or arterial hypertension, which, per se, may alter autonomic nervous system control. Breast cancer survivors had no evidence of metastases, and none of them underwent chemotherapy or radiotherapy treatments during the study period. All patients did not modify endocrine therapy and/or HER-2-directed therapies or other therapies throughout the study period.

Informed consent was obtained from all individuals participating in the study. The protocol of this study followed the principles of the Declaration of Helsinki and Title 45, US Code of Federal Regulations, Part 46, Protection of Human Subjects, Revised 13 November 2001, effective 13 December 2001, and was approved by the local Institutional Ethics Committee (letter signed by Humanitas Independent Ethics Committee 13th October 2015). All participants at the time of first clinical assessment signed an agreement to use anonymized data for population studies and possible publications.

To maximize time and cost effectiveness, the protocol was limited to sequentially planned outpatient office visits, as per good clinical practice. Subjects were evaluated at the first encounter (T0) and at the last planned encounter (T1) (after 17.63 \pm 11.4 months). Visits were scheduled every 2-3 months; the first one lasted $45 \mathrm{~min}$ while the subsequent ones lasted $30 \mathrm{~min}$.

In our clinic, patients, after around six months, lose at least $5 \%$ of their initial weight and continue the program on their own [32]. Nevertheless, we offer them the possibility to be followed for a further period. In this study, we consider subjects who, on a voluntary basis, elected to be followed for a long time. Throughout the entire intervention program, all patients were assessed and followed by the same physician, who had specific certified training in internal medicine (focusing on lifestyle changes) and clinical psychology. The protocol did not require the intervention of other professionals. Whenever deemed necessary, we gave patients some written material (for instance, nutritional tables).

Table 1 schematically describes first and follow-up visits, itemizing the multiple elements that were addressed, the time allotted and specific goals.

Table 1. Description of first and follow-up visits.

\begin{tabular}{|c|c|c|}
\hline $\begin{array}{l}\text { 1st Visit } \\
\text { Actions }\end{array}$ & Allotted Time & Goals \\
\hline Welcome & $5 \mathrm{~min}$ & $\begin{array}{l}\text { - Understand patient's concerns and circumstances } \\
\text { - Introduce the program and define patient's and physician's roles and responsibilities } \\
\text { - Establish an empathic, maieutic relationship }\end{array}$ \\
\hline $\begin{array}{c}\text { Clinical history and clinical } \\
\text { assessment }\end{array}$ & $10 \mathrm{~min}$ & $\begin{array}{l}\text { - Definition of patient's health status } \\
\text { - Take note of pharmacological plan and discuss potential side effects } \\
\text { - Discussion of lifestyle assessment (ad hoc questionnaire was filled in before encounter with physician) } \\
\text { - Collection of anthropometric data } \\
\text { - If required, prescription of further clinical tests }\end{array}$ \\
\hline $\begin{array}{l}\text { Explanation of diagnosis } \\
\text { and of specific benefits } \\
\text { derived from } \\
\text { lifestyle change }\end{array}$ & $5 \mathrm{~min}$ & $\begin{array}{l}\text { - Make the patient aware about her clinical condition } \\
\text { - Clear up facts from patient's interpretation of the personal implications } \\
\text { - Support the patient in learning how lifestyle change may ameliorate her health and reduce } \\
\text { cardiometabolic/oncologic risk, using her history } \\
\text { - Allow the patient to elicit desire, motivation to change } \\
\text { - Give the opportunity to the patient to raise any questions in order to have tailored answers }\end{array}$ \\
\hline $\begin{array}{l}\text { Setting of specific } \\
\text { individual goals }\end{array}$ & $5 \mathrm{~min}$ & $\begin{array}{l}\text { - Allow the patient to express her own reasons for change } \\
\text { - Assure the patient that she will have all the required support } \\
\text { - Prioritize behavior/issues for change (patient-physician alliance) } \\
\text { - Define long-term goal/s and steps (short-term goals) to reach it/them }\end{array}$ \\
\hline $\begin{array}{l}\text { Education about nutrition } \\
\text { and definition of tailored } \\
\text { nutritional plan }\end{array}$ & $10 \mathrm{~min}$ & $\begin{array}{l}\text { - Provide information about food choice } \\
\text { - Support the patient in discovering how to improve nutrition based on her/his history, clinical status and } \\
\text { lifestyle assessment } \\
\text { - Create a personalized nutrition program that the patient considers realistic, tailoring general clinical } \\
\text { guidelines to personalized actions } \\
\text { - Elicit in the patient the desire to adhere to the tailored program, taking into account medical guidelines, } \\
\text { personal clinical needs and patient's preferences }\end{array}$ \\
\hline $\begin{array}{l}\text { Education about physical } \\
\text { activity and tailored } \\
\text { exercise prescription }\end{array}$ & $10 \mathrm{~min}$ & $\begin{array}{l}\text { - Provide information about exercise } \\
\text { - Be sure that the patient understands the importance of reducing sedentary behaviors and of increasing } \\
\text { structured exercise } \\
\text { - Help the patient to discover and define practical strategies to reduce sedentariness during her normal } \\
\text { daily activities }\end{array}$ \\
\hline
\end{tabular}


Table 1. Cont.

\begin{tabular}{|c|c|c|}
\hline $\begin{array}{l}\text { Follow-Up Visit } \\
\text { Actions }\end{array}$ & Allotted Time & Goals \\
\hline Welcome & $1 \mathrm{~min}$ & - Reinforce the empathic, maieutic relationship \\
\hline $\begin{array}{l}\text { Clinical history (from } \\
\text { previous encounter to } \\
\text { present) and clinical } \\
\text { assessment }\end{array}$ & $9 \mathrm{~min}$ & $\begin{array}{l}\text { - Assess changes in clinical status and lifestyle from previous visit to present } \\
\text { - Collection of present anthropometric data } \\
\text { - Discussion of tests results prescribed during or after first visit }\end{array}$ \\
\hline $\begin{array}{l}\text { Analysis of results and of } \\
\text { encountered barriers }\end{array}$ & $5 \mathrm{~min}$ & $\begin{array}{l}\text { - Provide feedback on progression towards set goals } \\
\text { - Empower the patient and reflect on her central role in determining her health status and the success of } \\
\text { the lifestyle change program } \\
\text { - Reinforce the collaborative relationship with the physician and the possibility to get all the } \\
\text { required support } \\
\text { - Make the patient aware of the (even small) result/s obtained and how it/them has/have improved her } \\
\text { quality of life }\end{array}$ \\
\hline Problem solving & $10 \mathrm{~min}$ & $\begin{array}{l}\text { - Help the patient to find solution to overcome the encountered problems } \\
\text { - Help the patient to discover the required resources and to select those which best account for her needs } \\
\text { - Raise patient awareness of consequence of her/his behavior on her/his quality of life and wellbeing } \\
\text { - Make the patient aware that to encounter barriers and to find the resources to overcome them is a } \\
\text { critical, always present, part of a lifestyle change program }\end{array}$ \\
\hline
\end{tabular}

\subsection{Clinical Assessment (Necessary Also to Exclude Any Contraindication to Exercise)}

Clinical assessment comprised the following:

- History, evaluation of previous medical tests, standard medical examination, anthropometric and hemodynamic data

- Blood tests (fasting plasma glucose; total, HDL (high density lipoproteins) and LDL (low density lipoproteins) cholesterol; triglycerides; blood count; ALT (alanine transaminase) and AST (aspartate transaminase) levels measured at T0 in all subjects and at $\mathrm{T} 1 \mathrm{in} 73 \%$ of subjects, due to economic constraints.

- BIA bioelectrical impedance analysis (BodyStat Quadscan 4000, BodystatR_Quadscan 4000, Body Stat Ltd., Isle of Man, British Isles) [33] was employed in order to estimate percentage of fat mass (FM) and of free fat mass (FFM) using the proprietary equation provided by the manufacturer.

\subsection{Cardiac Autonomic Regulation}

Our approach to the non-invasive evaluation of autonomic regulation has recently been summarized [34,35]. In brief, ECG, non-invasive (Finometer, TNO, Delft, The Netherlands) arterial pressure and respiratory activity (piezoelectric belt, Marazza, Italy) are acquired on a PC. Beat-by-beat data series of $5 \mathrm{~min}$ rest followed by $5 \mathrm{~min}$ upright data are analyzed offline with dedicated software that provides time and frequency domain indices of variability.

As we described, combining the three most informative indices (RR interval, RR VARiance and stand-rest difference in RRLowFrequency in normalized units), a unitary autonomic nervous system index (ANSI, autonomic nervous system index) is obtained [27]. ANSI, free of age and gender bias by design, is treated as a percent ranked (0-100) unitary proxy of cardiac autonomic regulation, whereby higher values indicate better autonomic function.

\subsection{Lifestyle Assessment}

After the visit, before the autonomic nervous system evaluation, an ad hoc questionnaire to quantify lifestyle [12,36] was employed:

- Physical activity (weekly physical activity volume) was assessed by a modified version of the commonly employed short version of the International Physical Activity Questionnaire [37,38], which focuses on intensity (nominally estimated in metabolic equivalents-MET-according to the type of activity) and duration (in minutes) of physical activity. We considered the following levels: brisk walking $(\approx 3.3 \mathrm{METs})$, other activities of moderate intensity $(\approx 4.0 \mathrm{METs})$ and activities of vigorous intensity ( $\approx 8.0$ METs). 
- Nutrition was assessed using the AHA Diet Score [6], taking into consideration fruit/vegetables, fish, sweetened beverages, whole grain and sodium consumption (the assessment of the latter was adapted to Italian eating habits using a single question inquiring as to the habits of eating sodium-rich foods or adding sodium to food [39,40].

- Perceptions of stress, fatigue, control and somatic symptoms (4SQ) were assessed using a self-administered questionnaire [12,36] providing nominal self-rated Likert scales from 0 ("no perception") to 10 ("highest perception") for each measure. The 4SQ considers 18 somatic symptoms; thus, the total score ranged from 0 to 180.

\subsection{Statistical Analysis}

Data are presented as mean \pm SD (standard deviation). Differences between data at $\mathrm{T} 0$ and $\mathrm{T} 1$ were assessed with paired statistics (either paired $\mathrm{T}$ test or Wilcoxon signed ranks test for non-normally distributed variables, as indicated by Kolmogorov-Smirnov test). Computations were performed with a commercial package (IBM SPSS 26) considering $p<0.05$ as the significance threshold.

\section{Results}

Table 2 shows anthropometric, metabolic, hemodynamic and behavioral parameters for the 26 patients that were followed for $17.63 \pm 11.4$ months.

Table 2. Anthropometric, hemodynamic and metabolic data, before and after lifestyle management program.

\begin{tabular}{cccc}
\hline Variables & Before & After & $\boldsymbol{t}$-Test \\
\hline Age (years) & $52.9 \pm 8.1$ & & \\
Gender (f/m) & $23 / 3$ & & \\
Smoke (no/yes) & $19 / 7$ & $19 / 7$ & \\
Height $(\mathrm{cm})$ & $162.2 \pm 8.5$ & & \\
Weight $(\mathrm{kg})$ & $74.9 \pm 18.6$ & $65.9 \pm 15.8$ & $<\mathbf{0 . 0 0 1}$ \\
BMI $\left(\mathrm{kg} / \mathrm{m}^{2}\right)$ & $28.1 \pm 5.7$ & $24.8 \pm 4.8$ & $<\mathbf{0 . 0 0 1}$ \\
Waist circumference (cm) & $96.1 \pm 14.8$ & $89.9 \pm 15.1$ & $<\mathbf{0 . 0 0 1}$ \\
Fat Mass (\%) & $37.4 \pm 7.5$ & $33.3 \pm 7.0$ & $<\mathbf{0 . 0 0 1}$ \\
SAP (mmHg) & $114.7 \pm 17.4$ & $111.5 \pm 14.5$ & 0.198 \\
DAP (mmHg) & $75.1 \pm 9.5$ & $69.8 \pm 10.2$ & $\mathbf{0 . 0 0 1}$ \\
HR (b/min) & $68.5 \pm 8.9$ & $62.7 \pm 9.1$ & $\mathbf{0 . 0 0 1}$ \\
DP ((b/min) $\times$ mmHg) & $7861.7 \pm 1593.0$ & $7016.4 \pm 1429.0$ & $\mathbf{0 . 0 0 1}$ \\
AHA Diet Score (a.u.) & $2.95 \pm 1.09$ & $3.40 \pm 0.88$ & $\mathbf{0 . 0 4 5}$ \\
ActivityVolume (MET.min/week) & $778.5 \pm 1290.7$ & $1439.8 \pm 1335.0$ & $<\mathbf{0 . 0 0 1}$ \\
SEDENTARINESS (h/week) & $38.2 \pm 21.8$ & $32.4 \pm 22.7$ & 0.250 \\
STRESS perception (au) & $5.70 \pm 3.45$ & $4.35 \pm 2.77$ & $\mathbf{0 . 0 3 4}$ \\
FATIGUE perception (au) & $6.45 \pm 2.37$ & $4.90 \pm 3.25$ & 0.103 \\
4SQ Score (au) & $58.0 \pm 29.5$ & $44.8 \pm 26.4$ & $\mathbf{0 . 0 0 5}$ \\
CONTROL perception (au) & $5.55 \pm 2.60$ & $7.35 \pm 2.08$ & 0.075 \\
Fasting glucose (mg/dL) & $94.1 \pm 9.4$ & $92.5 \pm 13.5$ & 0.642 \\
Total cholesterol (mg/dL) & $234 . \pm 29.1$ & $203.9 \pm 29.1$ & $\mathbf{0 . 0 3 6}$ \\
HDL cholesterol (mg/dL) & $58.2 \pm 12.1$ & $66.1 \pm 16.8$ & $\mathbf{0 . 0 3 5}$ \\
LDL cholesterol (mg/dL) & $133.4 \pm 29.0$ & $104.7 \pm 33.7$ & 0.256 \\
Triglycerides (mg/dL) & $104.6 \pm 33.8$ & $90.1 \pm 26.8$ & 0.169 \\
\hline
\end{tabular}

Data are presented as mean $\pm \mathrm{SD}$; significance according to paired $t$-test. Abbreviations: $\mathrm{BMI}=$ body mass index; $\mathrm{SAP}=$ systolic arterial pressure; $\mathrm{DAP}=$ diastolic arterial pressure; $\mathrm{HR}=$ heart rate; $\mathrm{DP}=$ double product; $\mathrm{AHA}=$ American Heart Association; MET = metabolic equivalent; $4 \mathrm{SQ}=$ Subjective Somatic Stress Symptoms Questionnaire; HDL = high density lipoprotein; $\mathrm{LDL}=$ low density lipoprotein.

Weight, BMI, waist circumference, fat mass $\%$ and absolute (in $\mathrm{kg}$ ) were all significantly reduced at T1. Notably, weight diminished by around $10.95 \%$ (SD 6.08). Total cholesterol was slightly reduced and HDL cholesterol was increased (notably, no treatment with statin was started after first evaluation). Hemodynamic and behavioral indices were also modified coherently: HR, arterial pressure and double 
product were reduced significantly; AHA diet score increased, as well as weekly activity volume, as estimated by metabolic equivalents (METs min/week); subjective stress and subjective somatic symptoms perception (4SQ) diminished significantly (Figure 1).
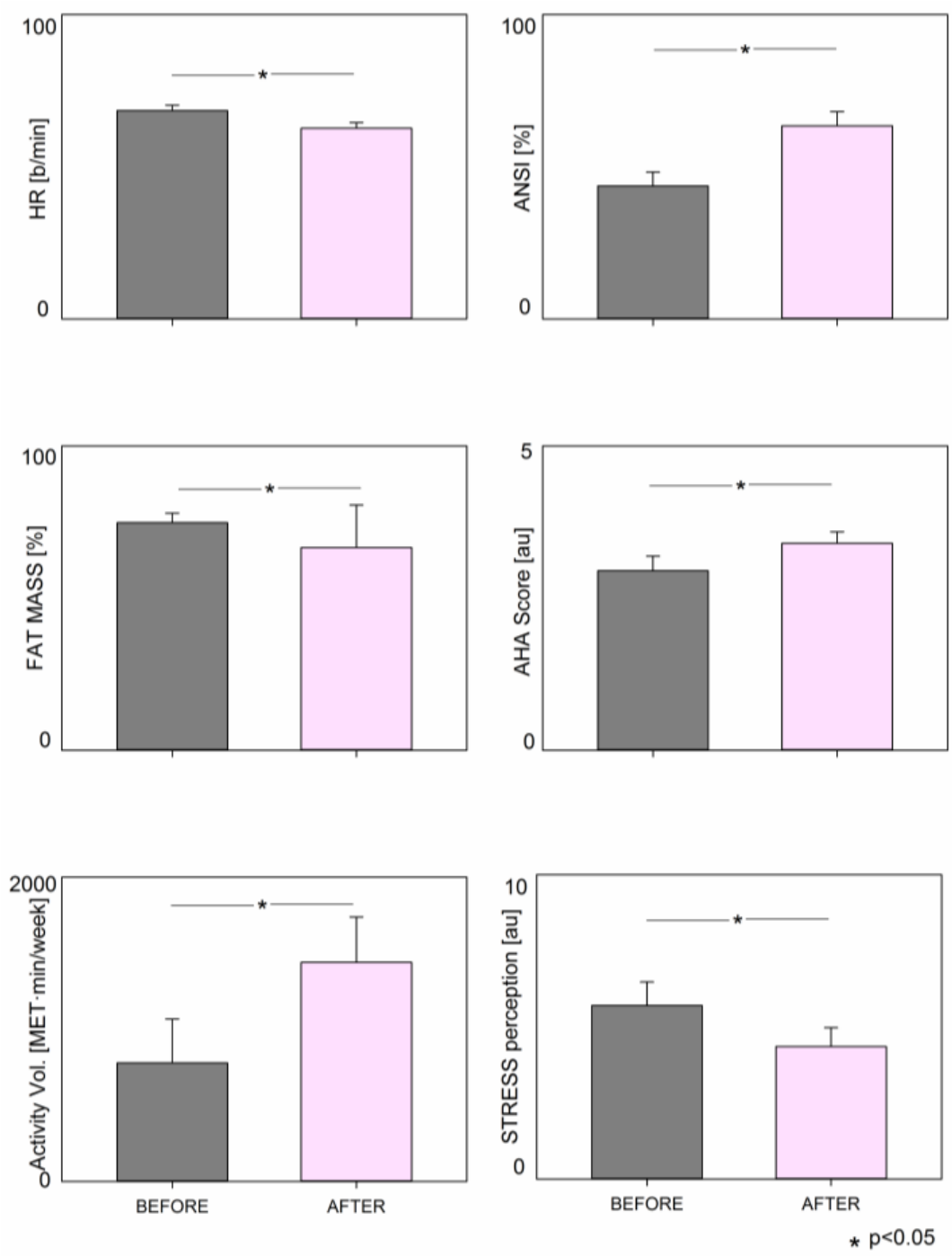

Figure 1. Schematic representation of the differences in selected metabolic, behavioral, hemodynamic and autonomic indices before and after lifestyle modification program in patients with increased cardiometabolic risk.

Table 3 and Figure 1 synthetically report the difference in autonomic indices between T0 and T1. In addition to an increase in RR, there are clear, significant increases in RR variance, RRHF in msec2 and, in ANSI, the composite index of cardiac autonomic regulation. Conversely, oscillatory indices (RR LFnu, RR HFnu and RRLF/HF), together with non-linear markers, were not different at T1 from T0. In addition, systolic arterial pressure (SAP) mean, SAPLFmmHg2 and indices of cardiac ( $\alpha$ index) or arterial baroreflex (A.XXAR) were not modified significantly. 
Table 3. Values of cardiac autonomic proxies before and after lifestyle management program.

\begin{tabular}{ccccc}
\hline Variable & & Before & After & $p$ \\
\hline RR & $\left(\mathrm{msec}^{2}\right.$ & $891.6 \pm 125.2$ & $977.4 \pm 156.1$ & $\mathbf{0 . 0 0 1}$ \\
RR VAR & $\left(\mathrm{msec}^{2}\right)$ & $1227.8 \pm 1053.1$ & $1727.8 \pm 1215.6$ & $\mathbf{0 . 0 0 8}$ \\
RR LFa & $\left(\mathrm{msec}^{2}\right)$ & $323.3 \pm 343.7$ & $559.8 \pm 880.9$ & 0.118 \\
RR HFa & $\left(\mathrm{msec}^{2}\right)$ & $312.9 \pm 382.9$ & $445.9 \pm 379.1$ & $\mathbf{0 . 0 0 7}$ \\
RR LFnu & $(\mathrm{nu})$ & $47.3 \pm 22.8$ & $45.4 \pm 24.9$ & 0.748 \\
RR HFnu & $(\mathrm{nu})$ & $45.7 \pm 21.9$ & $48.6 \pm 24.3$ & 0.621 \\
RR LF/HF & $()$. & $1.84 \pm 2.26$ & $2.25 \pm 3.39$ & 0.959 \\
RRRo & $(\mathrm{au})$ & $0.30 \pm 0.09$ & $0.29 \pm 0.09$ & 0.592 \\
RR $p \_0 \mathrm{v}$ & $(\%)$ & $23.0 \pm 13.7$ & $21.4 \pm 13.4$ & 0.623 \\
RR $p \_1 v$ & $(\%)$ & $48.7 \pm 6.9$ & $48.9 \pm 10.1$ & 0.921 \\
RR $p \_21$ & $(\%)$ & $10.9 \pm 7.1$ & $11.1 \pm 6.2$ & 0.948 \\
RR $p \_2 \mathrm{u}$ & $(\%)$ & $16.9 \pm 8.9$ & $18.4 \pm 9.8$ & 0.218 \\
A.XXAR & $\left(\mathrm{msec} / \mathrm{mmHg}^{2}\right)$ & $2.72 \pm 23.0$ & $2.58 \pm 2.46$ & 0.852 \\
SAP mean & $(\mathrm{mmHg})$ & $117.6 \pm 18.2$ & $114.5 \pm 21.0$ & 0.439 \\
SAP LFa & $(\mathrm{mmHg})$ & $3.5 \pm 4.2$ & $4.2 \pm 7.1$ & 0.675 \\
$\alpha$ Index & $\left(\mathrm{msec} / \mathrm{mmHg}^{2}\right)$ & $14.6 \pm 8.7$ & $16.2 \pm 8.9$ & 0.482 \\
ANSI & $(\%)$ & $43.7 \pm 23.0$ & $63.5 \pm 22.9$ & $\mathbf{0 . 0 0 1}$ \\
\hline
\end{tabular}

Abbreviations: $p=$ significance; RR = average of RR interval from tachogram sections; RR VAR $=$ variance from tachogram sections; $\mathrm{LFa}=$ absolute power(a) of low frequency (LF) component of RR variability; RR HFa = absolute power(a) of high frequency (HF) component of RR variability; RR LFnu = normalized power (nu) of low frequency (LF) component of RR variability; RR HFnu = normalized power $(\mathrm{nu})$ of high frequency (HF) component of RR variability; $\mathrm{RR} \mathrm{LF} / \mathrm{HF}=$ ratio between absolute values of $\mathrm{LF}$ and $\mathrm{HF}$; $\mathrm{Ro}=$ regularity index; $p_{-} 0 \mathrm{v}, p_{-} 1 \mathrm{v}, p_{-} 2 \mathrm{l}, p_{-} 2 \mathrm{v}=$ deterministic patterns; A.XXAR = index of arterial baroreflex; SAP mean = systolic arterial pressure by Finometer; $\alpha$ Index = index of overall cardiac baroreflex sensitivity; ANSI = Autonomic Nervous System Index

\section{Discussion}

This proof of concept study shows that it is possible to introduce a convenient cost effective lifestyle modification program into the currently existing clinical practice of secondary cardiometabolic prevention, capable of improving cardiac autonomic regulation in addition to body mass composition and stress perception.

\subsection{Cardiac Autonomic Regulation}

LMP represents a convenient strategy in the management of chronic non-communicable diseases $[3-7,10]$. Several studies based on structured, supervised interventions showed that improved metabolic effects can be considered an achievable goal $[6,7,10,13,41]$. The surprising finding that the attendant reduction of risk may be greater than expected from metabolic improvement alone suggested a significant beneficial role of improved autonomic regulation [18]. An impairment of CAR characterizes many common metabolic conditions, ranging from obesity and metabolic syndrome [18-23] to the side effects of multiple adjuvant therapies [26], increasing cardiac risk. In the present study, a significant improvement in CAR was observed after LMP, as clearly indicated by the increase in ANSI. Notably, among the multiple autonomic indices extracted from spectral analysis of RR interval variability and coding for different aspects of the variability phenotype (pulse, amplitude and oscillations), only the first two domains were favorably modified after the intervention. Oscillatory domain indices (RRLF and RRHF expressed in normalized units) conversely appeared unmodified by this LMP. The statistical procedure employed to build ANSI combines metrics of the three major domains of RR variability into a single, composite index of autonomic (sympathetic and parasympathetic) balance. In addition, ANSI is, by design, insensitive to age and gender and is presented as \% rank. Moreover, ANSI correlates with cardiac baroreflex sensitivity (generally considered an important index in determining cardiac prognosis) [42]. These properties render ANSI simple to interpret as a proxy of CAR, hence particularly useful and economical to gauge individually the results of LMP.

The observed improvement in CAR was associated with a significant reduction in fat mass (both in absolute and \% units), BMI and waist circumference. Of relevance, fat mass was obtained with a reliable, 
albeit indirect, method [33]. This method suggests also that, in the period of observation, lasting around 18 months, the dry lean mass was, notably, unmodified (from $10.6 \pm 3.4$ to $9.5 \pm 3.4 \mathrm{~kg}$; $14.7 \pm 3.5$ to $14.7 \pm 3.6 \%$ ). Although the accuracy of estimations of dry lean mass depends on a proprietary equation provided by the manufacturer, potentially fraught by an unknown bias, data suggest a well-balanced effect of the nutrition intervention and physical activity prescription, as documented by a reduced fat mass and maintained muscle mass.

\subsection{Lifestyle Modification Program (LMP)}

Here, we introduce a convenient LMP (combining cognitive behavioral strategies with patient-tailored prescription of nutrition and home-based exercise) $[1,6,7,32]$ into the currently existing clinical practice of secondary cardiometabolic prevention. We accommodated all elements of LMP (see Table 1) into relatively few encounters, avoiding increases in allotted time and costs, although first visits might require a longer time than usual general medicine outpatient practice [43]. All the components of the LMP were managed by a single physician, while the majority of LMPs consider the involvement of several different healthcare professionals. A multidisciplinary approach is an essential component of lifestyle interventions in order to be able to address all the clinical, psychological and technical aspects needed to help patients improve behavior $[1,6-9,11,13,28]$.

While, in research environments, it is easy to find diverse competent professionals who are actually capable of working in a truly collaborative, goal-oriented team, it is more difficult to find these teams in a regular clinical setting. In practice, it might happen that patients are managed by physicians that are not sufficiently trained and tend to simply refer patients to different healthcare professionals in a non-coordinated, inefficient way $[10,13,15]$. Conversely, the possibility to have a single physician trained in lifestyle medicine [1,44] (i.e., having all required kinds of expertise) might help to overcome this issue, as well as carry an obvious economic advantage and enhance work efficiency. There are still very few physicians trained in lifestyle medicine. However a growing number of studies indicate that training physicians in lifestyle medicine might be worthwhile [45,46], particularly considering that it has been estimated that lifestyle-based primary prevention might outperform by $4-5$ folds traditional drug-based secondary prevention [45]. Moreover, a lifestyle program based on the patient's active role [28] that is feasible outside the hospital environment (home-based), but under medical control, may be a bridge towards living a normal life again (particularly for cancer survivors) and toward introducing exercise and healthy nutrition into everyday routine. This approach would help to overcome a major drawback of structured rehabilitation programs that are considered by patients as part of "acute" (short-term) therapeutic strategies which are incapable of inducing long-term lifestyle modifications. In this study, patients were assessed after a long period $(17.63 \pm 11.4$ months), showing that they were capable of maintaining the modified lifestyle: they increased self-reported activity volume, improved AHA Diet score, suggesting healthier dietary habits, (see Table 2), and reduced perception of stress. The present findings therefore suggest that the cognitive-behavioral approach-in particular, considering motivational interviewing models-employed in this LMP appears promising. Obviously, the possibility to add other different healthcare professionals to a physician trained in lifestyle medicine would improve the quality of the program when economic and organizational barriers are not present. Nutritionists, exercise physiologists, physiotherapists, psychologists, etc., may have an important specific additional role, particularly when following up with the subject/patient over time, adjusting the program, designing new protocols, etc., then contributing to the eradication of interprofessional barriers constructed as a result of historical professional hierarchies and tensions [47].

Finally, the time slots allocated to individual elements of the LMP (see Table 1) may appear too short per se, but, thanks to the single physician model, lifestyle education is in practice spread across the entire visit. The preceding time, even if formally dedicated to other matters, may be used to introduce some personalized educational aspect regarding exercise/nutrition. For instance, when the physician sets, with the patient, the specific goal/s to be reached, they indicate also the modality of exercise specifically required to reach the set goal/s, thus anticipating some educational aspects (if the 
goal is to reduce fat mass and improve lean mass, for instance, the physician explains the difference between aerobic endurance exercise (required to reduce fat mass) and strength exercise (required to improve muscle mass)). Moreover, in the time dedicated to the education, the physician addresses specific aspects important to start the program. More information will be offered during the follow-up visits. Obviously, it may be very difficult to concentrate in a short time all the important information. This is possible only if the entire process is managed by only one trained physician. Moreover, the quality of the process depends on the physician's experience, as occurs in every medical endeavor.

\subsection{Limitations}

The study is based on indirect methods and provides only approximate quantification of CAR; however, ANSI proved capable of furnishing rather solid information about cardiac autonomic regulation in normal and pathological conditions. The number of patients is small (only 26). They were the patients who subsequently attended our clinic and who were free of chronic diseases, such as diabetes or arterial hypertension, which per se may alter autonomic nervous system control, who did not change or stop any pharmacological treatment during the study and who consented to be followed for a long period. In this proof of concept study, we do not have a control group: this is because the result that we reported derives from the (post hoc) observation of our clinical activity where, for ethical reasons, we may not exclude some patients from the intervention on lifestyle. Metabolic findings are based on a limited number of patients (about 73\%) because of economic limitations of this proof of concept observational study. Lastly, the proof of concept nature of this study could not, by design, consider a control untreated group, since it was based on self-selected patients who consulted our clinic in order to change their normal lifestyle introducing LMP.

\section{Conclusions}

We conclude that this preliminary, proof of concept study supports the hypothesis that it is possible to introduce a convenient time and cost effective LMP into the currently existing clinical practice of secondary cardiometabolic prevention. Indeed, findings suggest that a successful comprehensive behavior change, possibly facilitated by the simplified approach employed in this study, is capable of improving cardiac autonomic regulation in addition to body mass composition and stress perception. Considering the contributing role of altered cardiac autonomic regulation to cardiometabolic risk, this study corroborates the role of non-pharmacological strategies, based on lifestyle modification, to reduce the risk of cardiometabolic diseases. Applying the concept of sustainability (a term usually used to indicate the avoidance of the depletion of natural resources in order to maintain an ecological balance to grant to future generations enough planet resources) to the medical scenario, LMP may be considered a sustainable tool. In fact, they permit us to take action today (to foster a healthy lifestyle) to preserve a great good (health) which, otherwise, might disappear. Moreover, the prevention of chronic non-communicable diseases (thanks to healthy nutrition, physical exercise, cessation of smoking and stress management) is an important tool to grant benefits not only at the individual level but also at a global level. In this latter case, the benefits are also due to the possibility of saving economical resources that might be necessary in order to manage these chronic diseases.

Author Contributions: Conceptualization, D.L., W.G. and M.P.; data curation, D.L., M.M. and M.P.; investigation, D.L., M.M., W.G. and M.P.; methodology, D.L. and M.M.; project administration, D.L.; supervision, D.L.; validation, D.L., M.M., W.G. and M.P.; writing-review and editing, D.L., W.G. and M.P. All authors have read and agreed to the published version of the manuscript.

Funding: This research received no external funding.

Conflicts of Interest: The authors declare no conflict of interest. 


\section{References}

1. American College of Lifestyle Medicine. The American College of Lifestyle Medicine Standards. 2015. Available online: wwwlifestylemedicineorg/standards (accessed on 16 September 2020).

2. Willett, W.C. Balancing life-style and genomics research for disease prevention. Science 2002, 296, 695-698. [CrossRef] [PubMed]

3. Piepoli, M.F.; Villani, G.Q. Lifestyle modification in secondary prevention. Eur. J. Prev. Cardiol. 2017, 24 (Suppl. 3), 101-107. [CrossRef]

4. Piepoli, M.F.; Hoes, A.W.; Agewall, S.; Albus, C.; Brotons, C.; Catapano, A.L.; Cooney, M.T.; Corrà, U.; Cosyns, B.; Deaton, C.; et al. 2016 European Guidelines on cardiovascular disease prevention in clinical practice: The Sixth Joint Task Force of the European Society of Cardiology and Other Societies on Cardiovascular Disease Prevention in Clinical Practice (constituted by representatives of 10 societies and by invited experts) Developed with the special contribution of the European Association for Cardiovascular Prevention \& Rehabilitation (EACPR). Eur. Heart J. 2016, 37, 2315-2381. [PubMed]

5. Janssen, V.; De Gucht, V.; Dusseldorp, E.; Maes, S. Lifestyle modification programmes for patients with coronary heart disease: A systematic review and meta-analysis of randomized controlled trials. Eur. J. Prev. Cardiol. 2012, 20, 620-640. [CrossRef]

6. Lloyd-Jones, D.M.; Hong, Y.; Labarthe, D.; Mozaffarian, D.; Appel, L.J.; Van Horn, L.; Greenlund, K.; Daniels, S.; Nichol, G.; Tomaselli, G.F.; et al. Defining and setting national goals for cardiovascular health promotion and disease reduction. Circulation 2010, 121, 586-613. [CrossRef]

7. Spring, B.; Ockene, J.K.; Gidding, S.S.; Mozaffarian, D.; Moore, S.; Rosal, M.C.; Brown, M.D.; Vafiadis, D.K.; Cohen, D.L.; Burke, L.E.; et al. Better population health through behavior change in adults: A call to action. Circulation 2013, 128, 2169-2176. [CrossRef]

8. Stead, M.; Craigie, A.M.; Macleod, M.; McKell, J.; Caswell, S.; Steele, R.J.C.; Anderson, A.S. Why are some people more successful at lifestyle change than others? Factors associated with successful weight loss in the BeWEL randomised controlled trial of adults at risk of colorectal cancer. Int. J. Behav. Nutr. Phys. Act. 2015, 12, 87. [CrossRef]

9. Murray, J.; Craigs, C.; Hill, K.; Honey, S.; House, A. A systematic review of patient reported factors associated with uptake and completion of cardiovascular lifestyle behaviour change. BMC Cardiovasc. Disord. 2012, 12, 120. [CrossRef]

10. Milani, R.V.; Lavie, C.J. Health Care 2020: Reengineering Health Care Delivery to Combat Chronic Disease. Am. J. Med. 2015, 128, 337-343. [CrossRef]

11. Murphy, A.W.; Cupples, M.E.; Smith, S.M.; Byrne, M.; Newell, J. Effect of tailored practice and patient care plans on secondary prevention of heart disease in general practice: Cluster randomised controlled trial. BMJ 2009, 339, b4220. [CrossRef]

12. Lucini, D.; Riva, S.; Pizzinelli, P.; Pagani, M. Stress management at the worksite: Reversal of symptoms profile and cardiovascular dysregulation. Hypertension 2007, 49, 291-297. [CrossRef]

13. Rao, G.; Burke, L.E.; Spring, B.; Ewing, L.J.; Turk, M.; Lichtenstein, A.H.; Cornier, M.-A.; Spence, J.D.; Coons, M. New and emerging weight management strategies for busy ambulatory settings: A scientific statement from the American Heart Association endorsed by the Society of Behavioral Medicine. Circulation 2011, 124, 1182-1203. [CrossRef] [PubMed]

14. Graham, I.M.; Stewart, M.; Hertog, M.l.G. Cardiovascular Round Table Task Force. Factors impeding the implementation of cardiovascular prevention guidelines: Findings from a survey conducted by the European Society of Cardiology. Eur. J. Cardiovasc. Prev. Rehabilit. 2006, 13, 839-845. [CrossRef] [PubMed]

15. Friedberg, M.W.; Schneider, E.C.; Rosenthal, M.B.; Volpp, K.G.; Werner, R.M. Association between participation in a multipayer medical home intervention and changes in quality, utilization, and costs of care. JAMA 2014, 311, 815-825. [CrossRef] [PubMed]

16. Engel, B.T. Psychosomatic medicine, behavioral medicine, just plain medicine. Psychosom. Med. 1986, 48, 466-479. [CrossRef] [PubMed]

17. Ahn, A.C.; Tewari, M.; Poon, C.S.; Phillips, R.S. The clinical applications of a systems approach. PLoS Med. 2006, 3, e209. [CrossRef]

18. Joyner, M.J.; Green, D.J. Exercise protects the cardiovascular system: Effects beyond traditional risk factors. J. Physiol. 2009, 587, 5551-5558. [CrossRef] [PubMed] 
19. La Rovere, M.T.; Bigger, J.T., Jr.; Marcus, F.I.; Mortara, A.; Schwartz, P.J. Baroreflex sensitivity and heart-rate variability in prediction of total cardiac mortality after myocardial infarction. ATRAMI (Autonomic Tone and Reflexes after Myocardial Infarction) Investigators. Lancet 1998, 351, 478-484. [CrossRef]

20. Lucini, D.; Mela, G.S.; Malliani, A.; Pagani, M. Impairment in cardiac autonomic regulation preceding arterial hypertension in humans. Circulation 2002, 106, 2673-2679. [CrossRef]

21. Ondicova, K.; Mravec, B. Role of nervous system in cancer aetiopathogenesis. Lancet Oncol. 2010, 11, 596-601. [CrossRef]

22. Lucini, D.; Zuccotti, G.V.; Scaramuzza, A.; Malacarne, M.; Gervasi, F.; Pagani, M. Exercise might improve cardiovascular autonomic regulation in adolescents with type 1 diabetes. Acta Diabetol. 2013, 50, 341-349. [CrossRef] [PubMed]

23. Grassı, G.; Seravalle, G.; Colombo, M.; Bolla, G.; Cattaneo, B.M.; Cavagnini, F.; Mancia, G. Body weight reduction, sympathetic nerve traffic, and arterial baroreflex in obese normotensive humans. Circulation 1998, 97, 2037-2042. [CrossRef] [PubMed]

24. Soares-Miranda, L.; Sattelmair, J.; Chaves, P.; Duncan, G.E.; Siscovick, D.S.; Stein, P.K.; Mozaffarian, D. Physical activity and heart rate variability in older adults: The Cardiovascular Health Study. Circulation 2014, 129, 2100-2110. [CrossRef] [PubMed]

25. Lucini, D.; Milani, R.V.; Costantino, G.; Lavie, C.J.; Porta, A.; Pagani, M. Effects of cardiac rehabilitation and exercise training on autonomic regulation in patients with coronary artery disease. Am. Hear. J. 2002, 143, 977-983. [CrossRef]

26. Lucini, D.; Malacarne, M.; Oggionni, G.; Gatzmeier, W.; Santoro, A.; Pagani, M. Endocrine Adjuvant Therapy might Impair Cardiac Autonomic Regulation in Breast Cancer Survivors. Cardiol. Cardiovasc. Med. 2019, 3, 034-049. [CrossRef]

27. Sala, R.; Malacarne, M.; Solaro, N.; Pagani, M.; Lucini, D. A composite autonomic index as unitary metric for heart rate variability: A proof of concept. Eur. J. Clin. Investig. 2017, 47, 241-249. [CrossRef]

28. Rollnick, S.; Butler, C.C.; Kinnersley, P.; Gregory, J.; Mash, B. Motivational interviewing. BMJ 2010, 340, c1900. [CrossRef] [PubMed]

29. Stefani, L.; Klika, R.; Mascherini, G.; Mazzoni, F.; Lunghi, A.; Petri, C.; Petreni, P.; Di Costanzo, F.; Maffulli, N.; Galanti, G. Effects of a home-based exercise rehabilitation program for cancer survivors. J. Sports Med. Phys. Fit. 2019, 59, 846-852. [CrossRef]

30. American College of Sports Medicine; Riebe, D.; Ehrman, J.K.; Liguori, G.; Magal, M. ACSM's Guidelines for Exercise Testing and Prescription, 10th ed.; Lippincot Williams \& Wilkins: Philadelphia, PA, USA, 2018.

31. Mozaffarian, D. Dietary and Policy Priorities for Cardiovascular Disease, Diabetes, and Obesity. Circulation 2016, 133, 187-225. [CrossRef]

32. Lucini, D.; Vigo, C.; Malacarne, M.; Gatzemeier, W.; Pagani, M. Lifestyle changes as internal medicine. Eur. J. Intern. Med. 2017, 43, e40-e42. [CrossRef]

33. Benton, M.J.; Swan, P.D.; Schlairet, M.C.; Sanderson, S. Comparison of body composition measurement with whole body multifrequency bioelectrical impedance and air displacement plethysmography in healthy middle-aged women. Health Care Women Int. 2011, 32, 1068-1078. [CrossRef] [PubMed]

34. Lucini, D.; Solaro, N.; Pagani, M. Autonomic differentiation map: A novel statistical tool for interpretation of heart rate variability. Front. Physiol. 2018, 9, 401. [CrossRef] [PubMed]

35. Oggionni, G.; Spataro, A.; Pelliccia, A.; Malacarne, M.; Pagani, M.; Lucini, D. Left Ventricular Hypertrophy in World Class Elite Athletes Is Associated with Signs of Improved Cardiac Autonomic Regulation. Available online: https://journals.sagepub.com/doi/10.1177/2047487319830534 (accessed on 12 February 2019).

36. Lucini, D.; Solaro, N.; Lesma, A.; Gillet, V.B.; Pagani, M.; Dickerson, J.; Ivannikov, M. Health promotion in the workplace: Assessing stress and lifestyle with an intranet tool. J. Med. Internet Res. 2011, 13, e88. [CrossRef] [PubMed]

37. Craig, C.L.; Marshall, A.L.; Sjostrom, M.; Bauman, A.E.; Booth, M.L.; Ainsworth, B.E.; Pratt, M.; Ekelund, U.; Yngve, A.; Sallis, J.F.; et al. International physical activity questionnaire: 12-country reliability and validity. Med. Sci. Sports Exerc. 2003, 35, 1381-1395. [CrossRef]

38. Minetto, M.A.; Motta, G.; Gorji, N.E.; Lucini, D.; Biolo, G.; Pigozzi, F.; Portincasa, P.; Maffiuletti, N.A. Reproducibility and validity of the Italian version of the International Physical Activity Questionnaire in obese and diabetic patients. J. Endocrinol. Investig. 2017, 41, 343-349. [CrossRef] [PubMed] 
39. Lucini, D.; Zanuso, S.; Solaro, N.; Vigo, C.; Malacarne, M.; Pagani, M. Reducing the risk of metabolic syndrome at the worksite: Preliminary experience with an ecological approach. Acta Diabetol. 2015, 53, 63-71. [CrossRef]

40. Lucini, D.; Zanuso, S.; Blair, S.; Pagani, M. A simple healthy lifestyle index as a proxy of wellness: A proof of concept. Acta Diabetol. 2014, 52, 81-89. [CrossRef]

41. Lean, M.E.; Leslie, W.S.; Barnes, A.; Brosnahan, N.; Thom, G.; McCombie, L.; Peters, C.; Zhyzhneuskaya, S.; Al-Mrabeh, A.; Hollingsworth, K.G.; et al. Primary care-led weight management for remission of type 2 diabetes (DiRECT): An open-label, cluster-randomised trial. Lancet 2018, 391, 541-551. [CrossRef]

42. Solaro, N.; Malacarne, M.; Pagani, M.; Lucini, D. Cardiac baroreflex, HRV, and statistics: An interdisciplinary approach in hypertension. Front. Physiol. 2019, 10, 478. [CrossRef]

43. Chen, L.M.; Farwell, W.R.; Jha, A.K. Primary care visit duration and quality. Arch. Intern. Med. 2009, 169, 1866-1872. [CrossRef]

44. Lianov, L.; Johnson, M. Physician competencies for prescribing lifestyle medicine. JAMA 2010, $304,202$. [CrossRef] [PubMed]

45. Hivert, M.; Arena, R.; Forman, D.E.; Kris-Etherton, P.M.; McBride, P.E.; Pate, R.R.; Spring, B.; Trilk, J.; Van Horn, L.; Kraus, W.E. Medical training to achieve competency in lifestyle counseling: An essential foundation for prevention and treatment of cardiovascular diseases and other chronic medical conditions: A scientific statement from the American Heart Association. Circulation 2016, 134, e308-e327. [CrossRef] [PubMed]

46. Trilk, J.L.; Nelson, L.; Briggs, A.; Muscato, D. Including Lifestyle Medicine in Medical Education: Rationale for American College of Preventive Medicine/American Medical Association Resolution 959. Am. J. Prev. Med. 2019, 56, e169-e175. [CrossRef] [PubMed]

47. Gates, A.B.; Kerry, R.; Moffatt, F.; Ritchie, I.K.; Meakins, A.; Thornton, J.S.; Rosenbaum, S.; Taylor, A. Movement for movement: Exercise as everybody's business? Br. J. Sports Med. 2017, 51, 767-768. [CrossRef]

(C) 2020 by the authors. Licensee MDPI, Basel, Switzerland. This article is an open access article distributed under the terms and conditions of the Creative Commons Attribution (CC BY) license (http://creativecommons.org/licenses/by/4.0/). 